\title{
Long-term survival with unmethylated MGMT glioblastoma multiforme
}

\author{
Joana Savva-Bordalo, André Soares, Patrícia Rocha \\ Manuel Jácome, Joaquina Maurício, Rui Ferreira
}

\begin{abstract}
Introduction: Glioblastoma multiforme is the most aggressive tumor of the central nervous system. Despite advances in its management, overall prognosis remains poor, with a median survival time of less than one year. Good response to chemotherapy with temozolomide (TMZ) is usually associated with methylation of the promoter of the O(6)-methylguanine-DNA methyltransferase (MGMT) gene. Case Report: We describe here a patient with glioblastoma multiforme, who had good prognostic clinical features, age and performance status, but unmethylated MGMT promoter and who survived for eight years while treated with a multi-modal approach. Conclusion: Good
\end{abstract}

Joana Savva-Bordalo ${ }^{1}$, André Soares ${ }^{2}$, Patrícia Rocha ${ }^{3}$, Manuel Jácome ${ }^{4}$, Joaquina Maurício ${ }^{5}$, Rui Ferreira ${ }^{6}$

Affiliations: ${ }^{1,5}$ Department of Medical Oncology \& Cancer Epigenetics Group, Research Center; IPO-Porto, R, Dr. António Bernardino de Almeida, P 4200072 Porto, Portugal; ' ${ }^{2}$ Department of Radiotherapy; IPO-Porto, R. Dr. António Bernardino de Almeida, P 4200072 Porto, Portugal; ${ }^{3}$ Department of Genetics; IPO-Porto, R. Dr. António Bernardino de Almeida, P 4200072 Porto, Portugal; ${ }^{4}$ Department of Pathology; IPO-Porto, R. Dr. António Bernardino de Almeida, P 4200072 Porto, Portugal; ${ }^{6}$ Department of Neurosurgery; IPO-Porto, R. Dr. António Bernardino de Almeida, P 4200072 Porto, Portugal.

Corresponding Author: Joana Savva-Bordalo, IPO-Porto, Serviço de Oncologia Médica, R. Dr. António Bernardino de Almeida, P 4200072 Porto, Portugal; Phone: +351 22 5084000; Fax: 351225084001;

Email: joanasavva@gmail.com

Received: 30 January 2011

Accepted: 25 March 2011

Published: 01 July 2011 response to treatment, despite the absence of MGMT hypermethylation, suggests that other genetic or molecular factors may predict prognosis and therapeutic response in patients with glioblastoma multiforme.

Key words: Glioblastoma, O(6)-MethylguanineDNA Methyltransferase, Methylation, Temozolomide

$* * * * * * * * *$

Savva-Bordalo J, Soares A, Rocha P, Jácome M, Maurício J, Ferreira R. Long-term survival with unmethylated MGMT glioblastoma multiforme. International Journal of Case Reports and Images 2011;2(7):8-12.

\section{$* * * * * * * * *$}

doi:10.5348/ijcri-2011-07-43-CR-3

\section{INTRODUCTION}

The survival of patients with glioblastoma multiforme (GBM) can be improved significantly by radiotherapy (RT) with concomitant temozolomide (TMZ) chemotherapy, followed by six cycles of adjuvant TMZ [1]. This therapy is especially effective if the promoter of the O(6)-methylguanine-DNA methyltransferase (MGMT) gene is methylated [2]. Similarly, TMZ enhances survival in patients with MGMT hypermethylation who experience tumor recurrence, when compared with patients with an unmethylated promoter [3].

\section{CASE REPORT}

A 25-year-old man presented in October 2001 with 

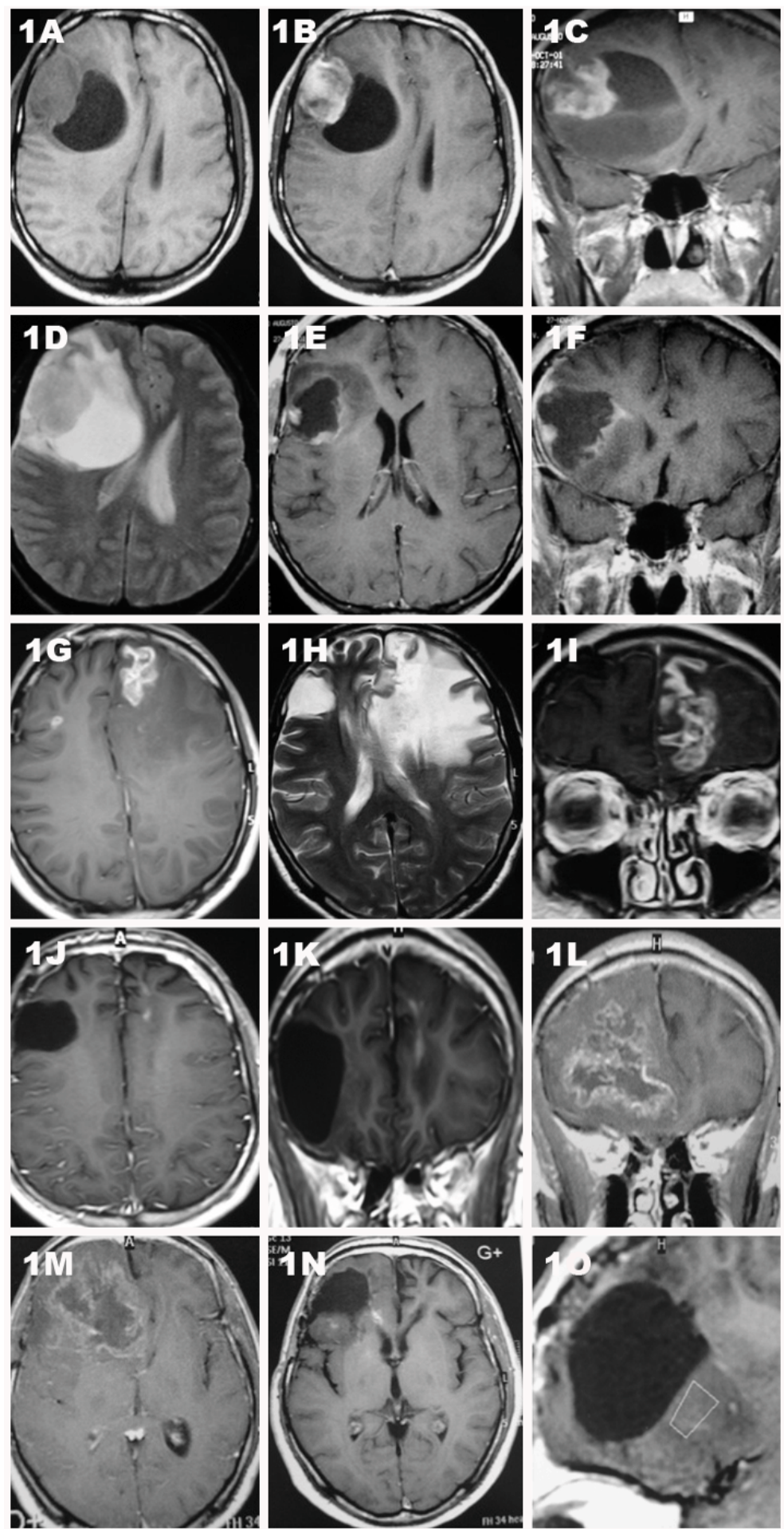

Figure 1: A) Gadolinium-enhanced axial pre-contrast T1-weighted, B) Axial post-contrast T1-weighted, C) Coronal postcontrast T1-weighted, D) Axial T2-weighted MRI images before the first operation, showing a right frontal-temporal-parietal lobe mass measuring $7 \times 6.5 \times 5 \mathrm{~cm}$, with a latero-superior solid component, a posterior cystic component and an inferior component with diffuse signal alteration of the encephalic parenchyma, suggesting a glial type tumor. E) Axial and F) Coronal pos-contrast T1-weighted postoperative MRI scan demonstrating contrast captation in a peripheral nodule, compatible with residual tumor. G) Axial pos-contrast T1-weighted, H) Axial T2-weighted and, I) Coronal pos-contrast T1-weighted images showing right residual tumor and a left paramedian frontal lesion, heterogeneously enhanced with gadolinium, invading the corpus callosum and collapsing the lateral left ventricle. There is also perilesional edema, mass effect on adjacent structures and midline shift. J, K) Post-contrast T1-weighted images after 14 cycles of temozolomide showing left residual enhancement and disappearance of the right lesion and surgical squeal. L, M) Post-contrast T1-weighted images before the second operation, suggesting tumor recurrence in the right frontal lobe, with a heterogeneous necrotic area and peripheral enhancement. N, O) Post-contrast T1-weighted with spectroscopy, evidencing residual tumor lesion, the encephaloclastic cavity on the right frontal lobe and post-radiation signs on the left frontal lobe. 


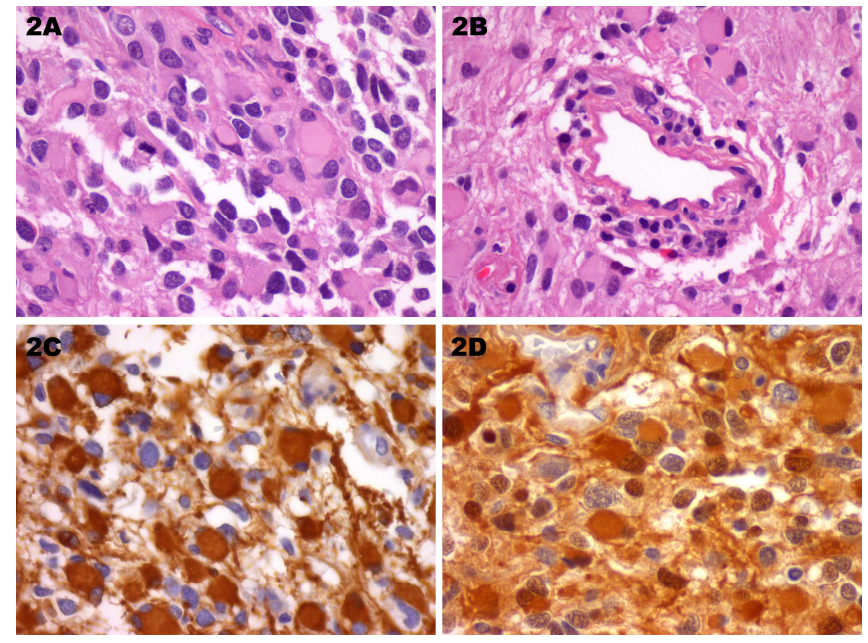

Figure 2: Histological specimens of the primary tumor (first surgery in 2001). A, B) Hematoxylin and eosin (H\&E) stained photomicrographs showing a typical glioblastoma multiforme with nuclear atypia, endothelial proliferation and hyperplastic blood vessels, which differentiate these tumors from Grade 3 astrocytomas, which do not have these features. C) Immunohistochemistry with antisera to glial fibrillary acidic protein (GFAP) and, D) S-100 protein showing strong labeling of tumor cells.

a constant headache of one month duration, which was localized in the frontal region and extended to the parietal area. The headache was accompanied by giddiness, photophobia and blurred vision. Nausea, vomiting and fever were not present. There were no precipitating, aggravating or relieving factors and the patient denied having any episodes of convulsive crisis or urinary incontinence. Despite the use of oral analgesics, headache had increased in intensity over time. The patient had no history of cranioencephalic trauma or previous exposure to ionizing radiation. With the exception of a 6 pack-year history of cigarette smoking, he did not present with any relevant personal or familial history.

On physical examination the patient was conscious, cooperative and orientated. He had excellent cognitive function, scoring more than 25 in the MMSE and a Karnofsky performance status of $100 \%$. The patient was apyretic and hemodynamically stable, with a rhythmic, ample and symmetric pulse. Cardiac and lung auscultation and abdominal examination were unremarkable. No rash or lymphadenopathy was noted. Nuchal rigidity was absent and cranial nerve test results were normal. Muscle strength was preserved bilaterally with normal deep tendinous and plantar cutaneous reflexes. There were no alterations on cerebellar testing or any sensory deficits. He had a normal complete blood cell count, renal and hepatic function, electrolytes, and coagulation results and was negative for inflammatory markers. Cranial magnetic resonance imaging (MRI) showed a right frontaltemporal-parietal lobe mass measuring 7x6.5 $55 \mathrm{~cm}$, suggesting a glial type tumor (Figures 1 A-D). The tumor was totally removed macroscopically in October 2001 (Figures 1 E, F); histopathologic examination revealed a WHO grade IV glioblastoma multiforme (GBM) (Figure 2). The patient received adjuvant RT (6o Gy in 6 weeks) and chemotherapy with carmustine (200 $\mathrm{mg} / \mathrm{m}^{2}$ intravenously every six weeks for six cycles).

The patient remained asymptomatic until August 2004, when he relapsed with a new left frontal lobe lesion and recurrence of the right surgical margins (Figures $1 \mathrm{G}-\mathrm{I}$ ). He was treated with 14 cycles of TMZ $\left(150 \mathrm{mg} / \mathrm{m}^{2} /\right.$ day for five days during each 28 day cycle), resulting in a very good partial response (Figures $1 \mathrm{~J}, \mathrm{~K}$ ).

In May 2008, he experienced a second tumor recurrence in the right frontal lobe (Figures $1 \mathrm{~L}-\mathrm{M}$ ). Subtotal removal was performed, confirming the diagnosis of GBM. Genomic DNA was isolated from paraffin-embedded tissue sections from the primary tumor and second recurrence. MGMT promoter methylation status was studied by MS-MLPA (Methylation specific multiplex ligation dependent probe amplification) (MLPA MRC-Holland), which detects methylation and copy number losses. No MGMT promoter methylation was found in any of the samples, but gene copy number loss was detected. In order to search for chromosome 10q deletion (the MGMT gene is located in 10q26), fluorescence in situ hybridization (FISH) analyses was performed in paraffin-embedded tissue sections, using a commercially available probe to target chromosome region 10q23 (ON PTEN-10q23/SE10; Kreatech diagnostics). There was no evidence of 10 23 (PTEN) deletion in any of the samples. Nevertheless, because of his previous good response to TMZ, the patient was again treated with TMZ $\left(150 \mathrm{mg} / \mathrm{m}^{2} /\right.$ day for 5 days in each 28 day cycle). He completed eight treatment cycles, resulting in a partial response (Figure $1 \mathrm{~N}, \mathrm{O}$ ) and remaining asymptomatic with residual imagiologic persistent disease. In April 2009, disease progression was observed. The tumor was again removed subtotally, reconfirming the former histological diagnosis. The patient was kept on symptomatic treatment thereafter and died in August 2009, eight years after his first diagnosis.

\section{DISCUSSION}

GBM is the most malignant type of astrocytic tumor, with a median survival time of less than one year [4]. Moreover, only $2 \%$ of patients with these tumors have unusually long survival after diagnosis [5]. Standard treatment involves a multimodal approach, combining maximum surgical resection, irradiation and chemotherapy [1]. Patient outcomes depend on the clinical, genetic and molecular characteristics of the tumor and on treatment choices.

Our patient first underwent surgical debulking, the 
preferred initial treatment to minimize neurological morbidity and enhance survival [6]. This was followed by RT and adjuvant chemotherapy with carmustine [7]. Since 2005, however, the standard treatment for patients with newly diagnosed GBM has consisted of surgery, RT plus concurrent TMZ followed by six cycles of adjuvant TMZ, a regimen that was found to improve survival appreciably when compared with RT alone [1]. Moreover, MGMT promoter methylation has been associated with significant improvements in survival following first line treatment [2]. At first recurrence the patient was treated with TMZ alone, which has been shown to be successful in patients with recurrence following primary treatment with surgery, RT and adjuvant nitrosourea chemotherapy [8-11]. Furthermore, in these patients, MGMT promoter methylation also predicts longer survival [3]. We found no MGMT promotor methylation in our patient, but instead a gene copy number loss, which is not a predictive factor for TMZ response [12]. Nevertheless, he achieved a very good partial response, lasting for four years after first relapse. Due to this long response, he was again treated with TMZ following the second recurrence, resulting in a partial response for 11 months.

Unlike most patients with GBM, who are older at presentation and survive less than one year, our patient was young and survived a total of eight years. Because our patient was young and had good performance status, treatment with multimodal therapy, consisting of surgical resection, RT, and adjuvant chemotherapy, resulted in longer survival, even though the $M G M T$ promoter was unmethylated.

Long-term GBM survivors (LTGBMS) may be biologically distinct from other patients with GBM [5]. Several other genomic changes have been reported in patients with GBM, including losses of heterozygosity of 1p, 9p, 10, 13q, 14q, 17p, 19p, and 22q loci and a gain of chromosome 7 [13]. These genetic alterations are responsible for some functional alterations, including the amplification, over expression, and/or mutation of growth factor receptors and loss of tumor suppressors (e.g. PTEN). However, the genetic and molecular features that can predict long-term survival are still unclear.

\section{CONCLUSION}

Our findings suggest that as yet unknown genetic and molecular features may contribute to long-term survival and therapeutic response in GBM.

$* * * * * * * *$

\section{ACKNOWLEDGEMENTS}

We thank Ana Isabel Salgado for reviewing brain imaging, Joana Vieira for the collaboration in the genetic analysis, António Verdelho and Machado
Carvalho for their suggestions and support.

\section{Author Contributions}

Joana Savva-Bordalo - Substantial contributions to conception and design, Acquisition of data, Analysis and interpretation of data, Drafting the article, Revising it critically for important intellectual content, Final approval of the version to be published

André Soares - Acquisition of data, Revising it critically for important intellectual content, Final approval of the version to be published

Patrícia Rocha, Manuel Jácome - Acquisition of data, Revising it critically for important intellectual content, Final approval of the version to be published

Joaquina Maurício - Acquisition of data, Revising it critically for important intellectual content, Final approval of the version to be published

Rui Ferreira - Acquisition of data, Revising it critically for important intellectual content, Final approval of the version to be published

\section{Guarantor}

The corresponding author is the guarantor of submission.

\section{Conflict of Interest}

Authors declare no conflict of interest.

\section{Copyright}

(C) Joana Savva-Bordalo et al. 2011; This article is distributed under the terms of Creative Commons attribution 3.0 License which permits unrestricted use, distribution and reproduction in any means provided the original authors and original publisher are properly credited. (Please see www.ijcasereportsandimages.com /copyright-policy.php for more information.)

\section{REFERENCES}

1. Stupp R, Mason WP, van den Bent MJ, Weller M, Fisher B, Taphoorn MJ, Belanger K, Brandes AA, Marosi C, Bogdahn U, et al: Radiotherapy plus concomitant and adjuvant temozolomide for glioblastoma. N Engl J Med. 2005;352(10):987-96.

2. Hegi ME, Diserens AC, Gorlia T, Hamou MF, de Tribolet N, Weller M, Kros JM, Hainfellner JA, Mason W, Mariani L, et al. MGMT gene silencing and benefit from temozolomide in glioblastoma. N Engl J Med. 2005;352(10):997-1003.

3. Sadones J, Michotte A, Veld P, Chaskis C, Sciot R, Menten J, Joossens EJ, Strauven T, D'Hondt LA, Sartenaer D, et al. MGMT promoter hypermethylation correlates with a survival benefit from temozolomide in patients with recurrent anaplastic astrocytoma but not glioblastoma. Eur $\mathrm{J}$ Cancer. 2009;45(1):146-53.

4. Brem SS, Bierman PJ, Black P, Brem H, Chamberlain MC, Chiocca EA, DeAngelis LM, Fenstermaker RA, Friedman A, Gilbert MR, et al. Central nervous system cancers. J Natl Compr Canc Netw. 2008;6(5):456-504. 
5. Senger D, Cairncross JG, Forsyth PA. Long-term survivors of glioblastoma: statistical aberration or important unrecognized molecular subtype? Cancer J. 2003;9(3):214-21.

6. DeVita Jr VTJ, Lawrence, Theodore S, Rosenberg, Steven A, DePinho, Ronald A, Weinberg, Robert A. Cancer: Principles and Practice of Oncology, Lippincott Williams \& Wilkins edn: Lippincott Williams \& Wilkins, 2008.

7. Gundersen S, Lote $\mathrm{K}$, Watne K. A retrospective study of the value of chemotherapy as adjuvant therapy to surgery and radiotherapy in grade 3 and 4 gliomas. Eur J Cancer. 1998;34(10):1565-9.

8. Perry JR, Rizek P, Cashman R, Morrison M, Morrison T. Temozolomide rechallenge in recurrent malignant glioma by using a continuous temozolomide schedule: the "rescue" approach. Cancer. 2008;113(8):2152-7.

9. Brandes AA, Ermani M, Basso U, Amista P, Berti F, Scienza R, Rotilio A, Pinna G, Gardiman M, Monfardini S. Temozolomide as a second-line systemic regimen in recurrent high-grade glioma: a phase II study. Ann Oncol. 2001;12(2):255-7.

10. Yung WK, Albright RE, Olson J, Fredericks R, Fink K, Prados MD, Brada M, Spence A, Hohl RJ, Shapiro W, et al. A phase II study of temozolomide vs. procarbazine in patients with glioblastoma multiforme at first relapse. $\mathrm{Br} \mathrm{J}$ Cancer. 2000;83(5):588-93.

11. Brada M, Hoang-Xuan K, Rampling R, Dietrich PY, Dirix LY, Macdonald D, Heimans JJ, Zonnenberg BA, Bravo-Marques JM, Henriksson R, et al. Multicenter phase II trial of temozolomide in patients with glioblastoma multiforme at first relapse. Ann Oncol. 2001;12(2):259-66.

12. Jeuken JW, Cornelissen SJ, Vriezen M, Dekkers MM, Errami A, Sijben A, Boots-Sprenger SH, Wesseling P. MS-MLPA: an attractive alternative laboratory assay for robust, reliable, and semiquantitative detection of MGMT promoter hypermethylation in gliomas. Lab Invest. 2007;87(10):1055-65.

13. Hu J, Pang JC, Tong CY, Lau B, Yin XL, Poon WS, Jiang CC, Zhou LF, Ng HK. High-resolution genome-wide allelotype analysis identifies loss of chromosome $14 \mathrm{q}$ as a recurrent genetic alteration in astrocytic tumours. Br J Cancer. 2002;87(2):21824. 